PROCEEDINGS OF THE

AMERICAN MATHEMATICAL SOCIETY

Volume 129, Number 10, Pages 2959-2963

S 0002-9939(01)06054-3

Article electronically published on March 29, 2001

\title{
COMPLEMENTED SUBSPACES OF PRODUCTS OF BANACH SPACES
}

\author{
ALEX CHIGOGIDZE
}

(Communicated by Nicole Tomczak-Jaegermann)

\begin{abstract}
We show that complemented subspaces of uncountable products of Banach spaces are products of complemented subspaces of countable subproducts.
\end{abstract}

\section{Introduction}

The following old unsolved problem (L. Nachbin 8]) of describing injective locally convex spaces is one of the general problems of the structure theory of locally convex spaces.

Problem 1. Is every injective locally convex space isomorphic to a product of Banach injective spaces?

In investigations related to this problem (see, for instance, 3], [1], [2], 4]) the following problem ([2, p. 71], [6, p. 147]) arose.

Problem 2. Is every complemented subspace of a product of a (countable) family of Banach spaces isomorphic to a product of Banach spaces?

G. Metafune and V. B. Moscatelli [5] p. 251] conjectured that this is false in general. Later this conjecture has been confirmed by M. Ostrovskii [9] who showed that not all complemented subspaces of countable products of Banach spaces are isomorphic to products of Banach spaces.

Our main result shows that for uncountable products the situation is somewhat different.

Theorem. A complemented subspace of an uncountable product of Banach spaces is a product of complemented subspaces of countable subproducts.

The following immediate corollary of this result provides a partial solution to Problem 1.

Corollary. Every injective locally convex space is isomorphic to a product of injective Fréchet spaces.

The author is grateful to P. Domańskii for his comments on the internet version of this note.

Received by the editors February 16, 2000.

1991 Mathematics Subject Classification. Primary 46A03, 46M10; Secondary 46A13.

Key words and phrases. Injective space, complemented subspace.

The author was partially supported by an NSERC research grant.

(C)2001 American Mathematical Society 


\section{RESUlts}

The following statement expresses a key fact used in the proof of Theorem 2.2

Let us first of all set up notation which will be used in the proofs of Proposition 2.1 and Theorem 2.2. For a subset $S \subseteq T$, where $T$ is an indexing set, let

$$
B_{S}=\prod\left\{B_{t}: t \in S\right\} \text { and } B=\prod\left\{B_{t}: t \in T\right\} .
$$

Also, for $S \subseteq R \subseteq T$, let $\pi_{S}: B \rightarrow B_{S}$ and $\pi_{S}^{R}: B_{R} \rightarrow B_{S}$ denote canonical projections onto the corresponding subproducts.

Proposition 2.1. Let $r: B \rightarrow B$ be a continuous linear map of an uncountable product of Banach spaces into itself. Also, let $A$ be a countable subset of $T$. Then there exist a countable subset $S$ of $T$ and a continuous linear map $r_{S}: B_{S} \rightarrow B_{S}$ such that $A \subseteq S$ and $\pi_{S} \circ r=r_{S} \circ \pi_{S}$.

Proof. Let $\exp _{\omega} T$ denote the set of all countable subsets of the indexing set $T$. Consider the following relation:

$$
\begin{gathered}
\mathcal{L}=\left\{(S, R) \in\left(\exp _{\omega} T\right)^{2}: S \subseteq R\right. \text { and there exists a continuous linear map } \\
\left.r_{S}^{R}: \prod\left\{B_{t}: t \in R\right\} \rightarrow \prod\left\{B_{t}: t \in S\right\} \text { such that } \pi_{S} \circ r=r_{S}^{R} \circ \pi_{R}\right\} .
\end{gathered}
$$

We need to verify the following three properties of the above defined relation.

(1) If $S \in \exp _{\omega} T$, then there exists $R \in \exp _{\omega} T$ such that $(S, R) \in \mathcal{L}$.

Proof. Since $B_{S}$ is a Fréchet space it follows that $\operatorname{ker}\left(\pi_{S} \circ r\right)$ is an intersection of countably many kernels of continuous seminorms. Consequently, $\pi_{S} \circ r$ factorizes through the projection $\pi_{R}$ for some countable $R$. Without loss of generality, we may assume that $S \subseteq R$. This shows that $(S, R) \in \mathcal{L}$.

(2) If $(S, R) \in \mathcal{L}, P \in \exp _{\omega} T$ and $R \subseteq P$, then $(S, P) \in \mathcal{L}$.

Proof. This is trivial because $\pi_{R}$ factorizes through $\pi_{P}$.

(3) Suppose that $\left(S_{i}, R\right) \in \mathcal{L}$ and $S_{i} \subseteq S_{i+1}$ for each $i \in \omega$. Then $\left(\bigcup\left\{S_{i}: i \in\right.\right.$ $\omega\}, R) \in \mathcal{L}$.

Proof. Consider the following projective sequence:

$$
\prod\left\{B_{t}: t \in S_{0}\right\} \stackrel{\pi_{S_{0}}^{S_{1}}}{\longleftarrow} \cdots \leftarrow \prod\left\{B_{t}: t \in S_{i}\right\} \stackrel{\pi_{S_{i}}^{S_{i+1}}}{\longleftarrow} \prod\left\{B_{t}: t \in S_{i+1}\right\} \leftarrow \cdots,
$$

the limit of which is isomorphic to the product $\prod\left\{B_{t}: t \in S\right\}$, where $S=$ $\bigcup\left\{S_{i}: i \in \omega\right\}$.

Since $\left(S_{i}, R\right) \in \mathcal{L}$, there exists a continuous linear map $r_{S_{i}}^{R}: \prod\left\{B_{t}: t \in R\right\} \rightarrow$ $\prod\left\{B_{t}: t \in S_{i}\right\}$ such that $\pi_{S_{i}} \circ r=r_{S_{i}}^{R} \circ \pi_{R}, i \in \omega$. Note that $\pi_{S_{i}}^{S_{i+1}} \circ r_{S_{i+1}}^{R}=r_{S_{i}}^{R}$ for each $i \in \omega$. Indeed, let $x \in \prod\left\{B_{t}: t \in R\right\}$ and consider any point $y \in \prod\left\{B_{t}: t \in\right.$ $T\}$ such that $x=\pi_{R}(y)$. Since $\left(S_{i}, R\right),\left(S_{i+1}, R\right) \in \mathcal{L}$ we have

$$
\begin{aligned}
\pi_{S_{i}}^{S_{i+1}}\left(r_{S_{i+1}}^{R}(x)\right) & =\pi_{S_{i}}^{S_{i+1}}\left(r_{S_{i+1}}^{R}\left(\pi_{R}(y)\right)\right)=\pi_{S_{i}}^{S_{i+1}}\left(\pi_{S_{i+1}}(r(y))\right) \\
& =\pi_{S_{i}}(r(y))=r_{S_{i}}^{R}\left(\pi_{R}(y)\right)=\pi_{S_{i}}^{R}(x) .
\end{aligned}
$$

In this situation the collection $\left\{\pi_{S_{i}}^{R}: \prod\left\{B_{t}: t \in R\right\} \rightarrow \prod\left\{B_{t}: t \in S_{i}\right\}: i \in \omega\right\}$ uniquely defines a continuous linear map $r_{S}^{R}: \prod\left\{B_{t}: t \in R\right\} \rightarrow \prod\left\{B_{t}: t \in S\right\}$ such that $\pi_{S_{i}}^{S} \circ r_{S}^{R}=r_{S_{i}}^{R}$ for each $i \in \omega\left(r_{S}^{R}\right.$ is simply the diagonal product of $r_{S_{i}}^{R}$ 's). 
It only remains to note that $\pi_{S} \circ r=r_{S}^{R} \circ \pi_{R}$ which completes the proof of the fact that $(S, R) \in \mathcal{L}$.

If $A \in \exp _{\omega} T$, then based on properties (1)-(3) we can inductively find an increasing sequence $\left\{S_{i}: i \in \omega\right\} \subseteq \exp _{\omega} T$ such that $A=S_{0}$ and $\left(S_{i}, S_{i+1}\right) \in \mathcal{L}$ for each $i \in \omega$. Clearly $S=\bigcup\left\{S_{i}: i \in \omega\right\}$ satisfies $(S, S) \in \mathcal{L}$. The proof is completed.

Theorem 2.2. A complemented subspace of a product of an uncountable family of Banach spaces is isomorphic to a product of Fréchet spaces. More formally, if $X$ is a complemented subspace of the product $\prod\left\{B_{t}: t \in T\right\}$ of Banach spaces $B_{t}, t \in T$, then $X$ is isomorphic to the product $\prod\left\{F_{j}: j \in J\right\}$, where $F_{j}$ is a complemented subspace of the product $\prod\left\{B_{t}: t \in T_{j}\right\}$ with $\left|T_{j}\right|=\omega$ for each $j \in J$.

Proof. Let $X$ be a complemented subspace of the product $B=\prod\left\{B_{t}: t \in T\right\}$, where $|T|=\tau>\omega$, and let $r: B \rightarrow X$ be a continuous projection. Let us agree that a subset $S \subseteq T$ is called $r$-admissible if $\pi_{S}(r(z))=\pi_{S}(z)$ for each point $z \in \pi_{S}^{-1}\left(\pi_{S}(X)\right)$.

Claim 1. The union of an arbitrary family of $r$-admissible sets is $r$-admissible.

Let $\left\{S_{j}: j \in J\right\}$ be a collection of $r$-admissible sets and $S=\bigcup\left\{S_{j}: j \in J\right\}$. Let $z \in \pi_{S}^{-1}\left(\pi_{S}(X)\right)$. Clearly $z \in \pi_{S_{j}}^{-1}\left(\pi_{S_{j}}(X)\right)$ for each $j \in J$ and consequently $\pi_{S_{j}}(r(z))=\pi_{S_{j}}(z)$ for each $j \in J$. Assuming that there is a point $z_{0} \in \pi_{S}^{-1}\left(\pi_{S}(X)\right)$ such that $\pi_{S}\left(r\left(z_{0}\right)\right) \neq \pi_{S}\left(z_{0}\right)$, we conclude that there exists an index $s \in S$ such that $\pi_{\{s\}}^{S}\left(\pi_{S}\left(r\left(z_{0}\right)\right)\right) \neq \pi_{\{s\}}^{S}\left(\pi_{S}\left(z_{0}\right)\right)$. Since $S=\bigcup\left\{S_{j}: j \in J\right\}$ it follows that there exists an index $j \in J$ such that $s \in S_{j}$. Then we have $\pi_{S_{j}}^{S}\left(\pi_{S}\left(r\left(z_{0}\right)\right)\right) \neq$ $\pi_{S_{j}}^{S}\left(\pi_{S}\left(z_{0}\right)\right)$. But this is impossible:

$$
\pi_{S_{j}}^{S}\left(\pi_{S}\left(r\left(z_{0}\right)\right)\right)=\pi_{S_{j}}(r(z))=\pi_{S_{j}}(z)=\pi_{S_{j}}^{S}\left(\pi_{S}\left(z_{0}\right)\right) .
$$

This contradiction proves the claim.

Claim 2. If $S \subseteq T$ is $r$-admissible, then $\pi_{S}(X)$ is a complemented subspace of $B_{S}=\prod\left\{B_{t}: t \in S\right\}$.

Indeed, let $i_{S}: B_{S} \rightarrow B$ be the canonical section of $\pi_{S}$ (this means that $i_{S}=$ $\left.\operatorname{id}_{B_{S}} \triangle \mathbf{0}: B_{S} \rightarrow B_{S} \times B_{T-S}=B\right)$. Consider a continuous linear map $r_{S}=\pi_{S} \circ r \circ$ $i_{S}: B_{S} \rightarrow \pi_{S}(X)$. Obviously, $i_{S}(y) \in \pi_{S}^{-1}\left(\pi_{S}(X)\right)$ for any point $y \in \pi_{S}(X)$. Since $S$ is $r$-admissible the latter implies that

$$
y=\pi_{S}\left(i_{S}(y)\right)=\pi_{S}\left(r\left(i_{S}(y)\right)\right)=r_{S}(y) .
$$

This shows that $\pi_{S}(X)$ is a complemented subspace of $B_{S}$.

Claim 3. Let $S$ and $R$ be $r$-admissible subsets of $T, S \subseteq R \subseteq T$. Then there exists an operator $g_{S}^{R}: X_{S} \rightarrow X_{R}$ such that $\pi_{S}^{R} \mid X_{R} \circ g_{S}^{R}=\operatorname{id}_{X_{S}}$.

Define $g_{S}^{R}=\pi_{R} \circ r \circ i_{S} \mid X$. By the proof of Claim 2, for $z \in \pi_{S}(X)$ we get $\pi_{S}^{R} \circ g_{S}^{R}(z)=\pi_{S} \circ r \circ i_{S}(z)=z$.

Claim 4. Every countable subset of $T$ is contained in a countable $r$-admissible subset of $T$. 
Let $A$ be a countable subset of $T$. Our goal is to find a countable $r$-admissible subset $S$ such that $A \subseteq S$. By Proposition [2.1, there exist a countable subset $S$ of $T$ and a continuous homomorphism $r_{S}: B_{S} \rightarrow B_{S}$ such that $A \subseteq S$ and $\pi_{S} \circ r=r_{S} \circ \pi_{S}$. Consider a point $y \in \pi_{S}(X)$. Also pick a point $x \in X$ such that $\pi_{S}(x)=y$. Then

$$
y=\pi_{S}(x)=\pi_{S}(r(x))=r_{S}\left(\pi_{S}(x)\right)=r_{S}(y) .
$$

This shows that $r_{S} \mid \pi_{S}(X)=\mathrm{id}_{\pi_{S}(X)}$ (this shows, in fact, that $\pi_{S}(X)$ is complemented in $B_{S}$ ).

In order to show that $S$ is $r$-admissible let us consider a point $z \in \pi_{S}^{-1}\left(\pi_{S}(X)\right)$. By the observation made above, $r_{S}\left(\pi_{S}(z)\right)=\pi_{S}(z)$. Finally,

$$
\pi_{S}(z)=r_{S}\left(\pi_{S}(z)\right)=\pi_{S}(r(x))
$$

which implies that $S$ is $r$-admissible.

We now use the above listed properties of $r$-admissible subsets and proceed as follows. By Claim 4, each element $t_{\alpha} \in T$ is contained in a countable $r$-admissible subset $S_{\alpha} \subseteq T$. According to Claim 1, the set $T_{\alpha}=\bigcup\left\{S_{\beta}: \beta \leq \alpha\right\}$ is $r$-admissible for each $\alpha<\tau$. Consider the projective system

$$
\mathcal{S}_{X}=\left\{X_{\alpha}, p_{\alpha}^{\alpha+1}, \tau\right\}
$$

where

$$
X_{\alpha}=\pi_{T_{\alpha}}(X) \text { and } p_{\alpha}^{\alpha+1}=\pi_{T_{\alpha}}^{T_{\alpha+1}} \mid \pi_{T_{\alpha+1}}(X): X_{\alpha+1} \rightarrow X_{\alpha} \text { for each } \alpha<\tau .
$$

Since $T=\bigcup\left\{T_{\alpha}: \alpha<\tau\right\}$, it follows that $X=\operatorname{proj} \lim \mathcal{S}$. Obvious transfinite induction based on Claim 3 shows that

$$
X=\operatorname{proj} \lim \mathcal{S}=X_{0} \times \prod\left\{\operatorname{ker}\left(p_{\alpha}^{\alpha+1}\right): \alpha<\tau\right\} .
$$

Since, by the construction, $S_{\alpha}$ is a countable $r$-admissible subset of $T$, it follows from Claim 2 that $X_{0}$ and $\operatorname{ker}\left(p_{\alpha}^{\alpha+1}\right), \alpha<\tau$, being complemented subspaces of countable products of Banach spaces, are Fréchet spaces. This finishes the proof of Theorem 2.2.

Recall that an object $X$ of the category $\mathcal{L} S C$ of locally convex spaces and their continuous linear maps is injective if any continuous linear map $f: A \rightarrow X$, defined on a linear subspace of a space $B$, admits a continuous linear extension $g: B \rightarrow X$ (i.e. $g \mid A=f$ ).

The following statement is related to Problem 1 stated in the Introduction.

Corollary 2.3. The following conditions are equivalent for a locally convex topological vector space $X$ :

(1) $X$ is an injective object of the category $\mathcal{L S C}$.

(2) $X$ is isomorphic to the product $\prod\left\{F_{t}: t \in T\right\}$, where each $F_{t}, t \in T$, is a complemented subspace of a product $\prod\left\{\ell_{\infty}\left(J_{t_{n}}\right): n \in \omega\right\}$.

Proof. (2) $\Longrightarrow(1)$. By [3] Lemma 0] and [7, p.105], $\ell_{\infty}(J)$ is an injective object of the category $\mathcal{L C S}$ for any set $J$. Obviously (see, for instance, 3, Lemma 1.9]), the product of an arbitrary collection of injective objects of the category $\mathcal{L C S}$ is also an injective object of this category. Consequently, the Fréchet space $F_{t}, t \in T$, as a complemented subspace of $\prod\left\{\ell_{\infty}\left(J_{t_{n}}\right): n \in \omega\right\}$, is injective. Finally, the space $X$, as a product of injectives, is an injective object of the category $\mathcal{L} \mathcal{S C}$. 
$(1) \Longrightarrow(2)$. Clearly $X$ can be embedded into $\prod\left\{\ell_{\infty}\left(J_{t}\right): t \in T\right\}$ for suitable $J_{t}$ and $T$. The required conclusion follows from Theorem 2.2 .

\section{REFERENCES}

[1] P. Domański, Complemented subspaces of products of Hilbert spaces, Proc. Amer. Math. Soc. 110 (1990), 187-196. MR 90m:46007

[2] _ $\mathcal{L}_{p}$-spaces and injective locally convex spaces, Diss. Math. 298 (1990), 1-76. MR 91j:46002

[3] P. Domański, A. Ortyński, Complemented subspaces of products of Banach spaces, Trans. Amer Math. Soc. 316 (1989), 215-231. MR 90b:46004

[4] G. Metafune, V. B. Moscatelli, Complemented subspaces of sums and products of Banach spaces, Ann. Mat. Pura Appl. 153 (1989), 1-16. MR 90j:46003

[5] , Quojection and prequojections, Advances in the Theory of Fréchet Spaces (T.Terzioğlu, ed.), Kluwer Academic Publishers, Dordrecht, 1989, pp. 235-254. MR 92e:46006

[6] On twisted Fréchet and (LB)-spaces, Proc. Amer. Math. Soc. 108 (1990), 145-150. MR 90d: 46007

[7] J. Lindenstrauss, L. Tzafriri, Classical Banach Spaces, I, Springer-Verlag, Berlin, 1977. MR 58:17766

[8] L. Nachbin, Some problems in extending and lifting continuous linear transformations, Proc. Internat. Sympos. Linear Spaces (Jerusalem, 1960), Jerusalem Academic Press, 1961, pp. 340350. MR 24:A2826

[9] M. Ostrovskii, On complemented subspaces of sums and products of Banach spaces, Proc. Amer. Math. Soc. 124 (1996), 2005-2012. MR 96i:46002

Department of Mathematics and Statistics, University of Saskatchewan, McLean Hall, 106 Wiggins Road, Saskatoon, Saskatchewan, Canada S7N 5 E6

E-mail address: chigogid@math.usask.ca 